\title{
EXPERIENCIA DE EVALUACIÓN FORMATIVA EN UNA ASIGNATURA DEL GRADO EN ENFERMERÍA
}

Experience of formative assessment in a nursing degree subject

Experiência de avaliação formativa em uma disciplina da licenciatura em enfermagem

\author{
Ana I. López-Alonso (1) \\ Leticia Sánchez-Valdeón (2) \\ Ma Concepción González-García (3) \\ Cristina Liébana-Presa (4) \\ Ana Ma Vázquez-Casares (5) \\ Ma Elena Fernández-Martínez (6)
}

(1) Universidad de León, España. Teléfono: +34 987293106. Correo electrónico: ana.lopez@unileon.es

(2) Universidad de León, España. Correo electrónico: $\underline{\text { lsanv@unileon.es }}$

(3) Universidad de León, España. Correo electrónico: mgonzg@unileon.es

(4) Universidad de León, España. Correo electrónico: cristina.liebana@unileon.es

(5) Universidad de León, España. Correo electrónico: ana.vazquez@unileon.es

(6) Universidad de León, España. Correo electrónico: elena.fernandez@unileon.es

\section{Resumen}

Se presenta la experiencia de incluir la evaluación formativa, empleada para la competencia “aplicar el Proceso de Enfermería para proporcionar y garantizar el bienestar, la calidad y seguridad a las personas atendidas”, que se está realizando en la asignatura de Enfermería del Adulto, de Grado en Enfermería de la Universidad de León, durante el curso 2016-2017. El trabajo refleja las actividades diversas y multimétodo de los procesos de evaluación llevados a cabo, tanto a nivel de adquisición de conocimientos, metodología científica aplicada, como en las prácticas de laboratorio. Incluye los instrumentos utilizados y posterior evaluación de los estudiantes. Los resultados preliminares están siendo muy positivos tanto para los profesores como para los estudiantes y un buen reflejo de ello son los resultados del rendimiento académico que se están obteniendo y las valoraciones positivas de los procesos de formación. 
Palabras clave: Evaluación formativa; Educación Superior; Evaluación por Competencias; Enfermería

\begin{abstract}
We report the experience of including formative assessment in the subject of Adult Nursing, part of the Degree in Nursing at the University of León, during the academic year 2016-2017, for the competency “applying the nursing process to provide and ensure quality care and patient well-being and safety”. We describe the diverse, multimethod evaluation activities carried out with regard to knowledge acquisition, scientific methodology applied, laboratory practicals, the instruments employed and the subsequent student evaluation. The preliminary results are very positive for both teachers and students, and this is reflected for example in the academic results being obtained and the positive evaluations received of the training processes.
\end{abstract}

Keywords: formative assessment; higher education; competency evaluation; nursing

\title{
Resumo
}

Apresenta-se a experiência de se incluir a avaliação formativa, usada na competência “aplicação do processo de enfermagem para fornecer e garantir o bem-estar, qualidade e segurança das pessoas atendidas”, que está sendo realizado na disciplina Enfermagem do Adulto, da Licenciatura em Enfermagem da Universidade de León, durante o ano letivo 2016/17. O trabalho reflete as diferentes atividades e o multimétodo dos processos de avaliação, realizados tanto na aquisição de conhecimentos, metodologia científica aplicada, como nas práticas de laboratório. O estudo inclui os instrumentos usados e a posterior avaliação dos alunos. Os resultados preliminares do estudo são positivos tanto para os professores como para os alunos. Um bom reflexo disso são os resultados acadêmicos obtidos e as avaliações satisfatórias dos processos educativos.

Palavras-chave: Avaliação formativa; Educação Superior; Avaliação por competências; Enfermagem

\section{Introducción}

Las competencias de las asignaturas del Grado de Enfermería aspiran a simular el ámbito profesional a través de una vinculación estrecha con lo que se realiza en la "vida real” (Valverde, Revuelta \& Fernández, 2012). La evaluación por competencias ofrece 
nuevas oportunidades a profesores y a estudiantes; los primeros, al generar entornos significativos de aprendizaje con experiencias académicas cercanas al mundo profesional, favorecen el aprendizaje centrado en los estudiantes, y estos desarrollan las capacidades de forma integrada y orientadas a la acción, es decir, se capacitan en la resolución de problemas prácticos al enfrentarse a situaciones “auténticas” (Palés \& Gomar, 2010; Valverde et al., 2012). Estas competencias están compuestas por un conjunto de estructuras de conocimiento, habilidades cognitivas y afectivas, actitudes y valores, necesarios para ejecutar tareas, solucionar problemas y desempeñar eficazmente la profesión (Wesselink, Lans, Mulder \& Biemans, 2003).

En este sentido, la evaluación formativa, al integrar en la evaluación las evidencias de aprendizaje y el nivel de logro de resultados a través de actividades diversas y multimétodo, incluyendo actitudes y valores (Valverde et al., 2012), consigue que profesores y estudiantes objetiven el proceso de aprendizaje.

Este trabajo pretende mostrar las actividades y herramientas de evaluación formativa que se han diseñado en la asignatura de Enfermería de Adulto, con las que se evalúa el aprendizaje del estudiante en diversos momentos, empleando TICs, Moodle, autoevaluación y coevaluación. El objetivo es incluir la evaluación formativa en la competencia "aplicar el Proceso de Enfermería (PE), para proporcionar y garantizar el bienestar, la calidad y seguridad a las personas atendidas”.

\section{Contextualización}

Enseñanza Superior. Grado en Enfermería, $1^{\circ}$ curso, $2^{\circ}$ semestre, asignatura Enfermería Adulto I, 6 créditos distribuidos en teoría $(2,55)$, seminarios $(1,65)$ y prácticas $(1,8)$. Los estudiantes matriculados en el curso 2016-2017 son 104 y los profesores implicados en la docencia 8. De ellos, 2 son funcionarios, 3 asociados académicos y 3 asociados clínicos. La experiencia se pone en marcha buscando funcionalidad, equilibrio y motivación para los profesores, que en cada curso pueden ser diferentes, y para los estudiantes, pues, al incluir la evaluación como sistema de aprendizaje, reciben unificados los criterios e instrumentos de evaluación.

\section{Diseño y desarrollo}

La evaluación formativa se propone a partir de un Caso Clínico (CC), que el estudiante desarrolla utilizando los conocimientos que se imparten en la asignatura y empleando el 
PE. La aplicación de las actividades y herramientas de la evaluación formativa son progresivas y están en proceso de validación.

A partir del CC se desarrollan los contenidos teóricos y prácticos de la asignatura. El aprendizaje de los aspectos teóricos se refleja en las autoevaluaciones de seguimiento que se realizan semanalmente con 10 preguntas de respuesta múltiple sobre una batería de 30, y de una sola opción correcta. Además, el estudiante realiza un cuestionario de valoración, satisfacción y sugerencias de mejora, sobre cada actividad que se le imparte (teórica y práctica); para el desarrollo del PE se forman grupos de 5 estudiantes con la herramienta consulta de Moodle, donde, además, se les sube la documentación, bibliografía y TICs de terminología enfermera para su consulta; el seguimiento se realiza a través del foro y de tutorías personalizadas.

El trabajo en equipo se evalúa con una escala modificada del documento del Grupo IFAHE (Domínguez-Fernández, 2015), y una parrilla de reparto de nota grupal (Pérez-Pueyo, Hortigüela, Hernando-Garijo \& Barba-Martín, 2013).

El diseño de escenarios de simulación, junto a los criterios de reproducibilidad y evaluación de habilidades, nos permite el desarrollo de las prácticas de laboratorio, así como la evaluación de las habilidades que adquieren los estudiantes; finalmente, se realiza una evaluación global de satisfacción con la asignatura; para ello utilizamos un cuestionario adaptado, aún no publicado, de López-Aguado.

La sistemática utilizada en la formación metodológica es:

- Exposición en el aula de contenidos y propuesta de ejercicios individuales o grupales no presenciales.

- Exposición en el aula de la corrección de los ejercicios y, a partir de ella, establecimiento de los criterios de evaluación. En los ejemplos de corrección se aplica la rúbrica y la coevaluación, con la participación de estudiantes y tres profesores.

\section{Resultados}

Se ha completado la elaboración de un caso clínico, con sus criterios de evaluación, rúbrica y escala de valoración de trabajo grupal. 
Se ha realizado una experiencia de coevaluación a través de la rúbrica con parte del PE, que representamos en la Figura 1.

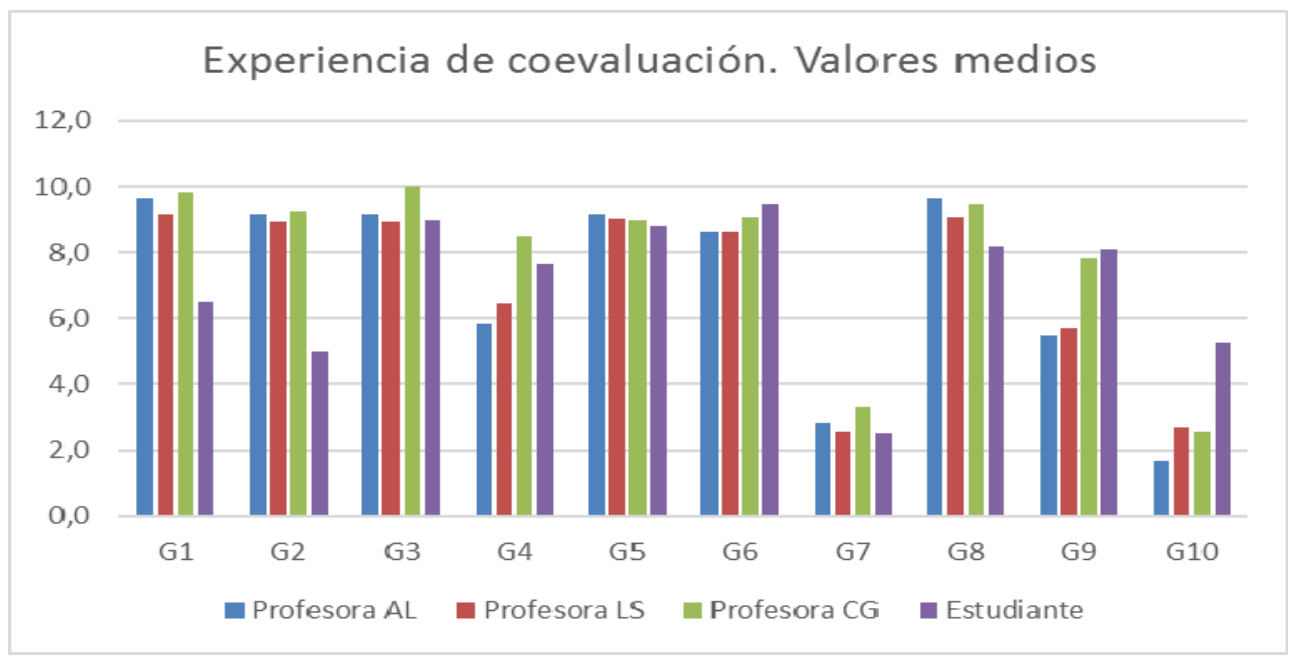

Figura 1.

Experiencia de coevaluación con la rúbrica (sobre 10)

Se han elaborado 8 cuestionarios de autoevaluación para los seminarios, 9 para las prácticas y sus respectivas valoraciones de satisfacción del estudiante. La tabla 1 expresa los resultados de las autoevaluaciones, y la figura 2 un ejemplo de valoración de satisfacción con un seminario.

Tabla 1.

Valores medios obtenidos en las autoevaluaciones (sobre 10)

\begin{tabular}{lll}
\hline $\mathbf{N}=\mathbf{1 0 2}$ & Seminarios & Prácticas \\
\hline Nota media & 7,2 & 8 \\
\hline
\end{tabular}

\section{Valoración del estudiante de la docencia impartida en el seminario de Cardiopatía Isquémica}

09.- Preparación final estudiantes

07. Motivación estudiantes

05. Atención prestada

03. Interés generado

01. Novedad contenidos

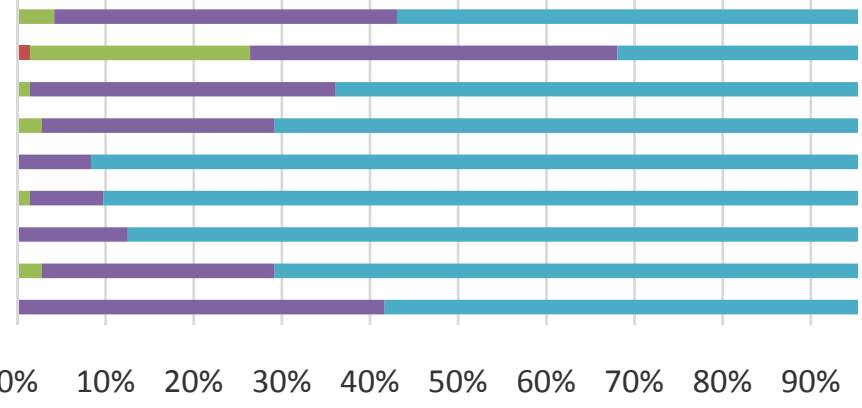

Figura2.

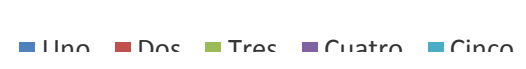

Valoraciones de satisfacción del estudiante

Experiencia de evaluación formativa en una asignatura del Grado en Enfermería 
Se han puesto en marcha las listas de verificación en 3 prácticas y se han realizado los videos de control de 9 de ellas

\section{Conclusiones}

Tanto las herramientas de evaluación formativa como las diferentes actividades propuestas están facilitando la implicación del profesorado y de los estudiantes en el proceso de aprendizaje competencial.

La información que comparten los profesores y estudiantes, sobre las actividades de la evaluación formativa, otorga transparencia al proceso de evaluación y herramientas objetivas para ambos.

A la vez, estas acciones compartidas motivan las tutorías frecuentes, donde se solucionan dudas sobre los procesos clínicos; este hecho hace que el estudiante afiance sus conocimientos y el profesor pueda evaluar su progresión.

Tanto las autoevaluaciones como la coevaluación, e incluso el mero conocimiento, por parte del estudiante, de la evaluación intragrupal (lo manifiestan en las tutorías), están siendo una motivación importante en su aprendizaje y para el trabajo grupal.

Finalmente, decir que se podría transferir esta experiencia a otras asignaturas del Grado en Enfermería, si bien sería preciso adaptarlo. La principal limitación en nuestro caso es el profesorado eventual que participa en la asignatura.

\section{Referencias}

Domínguez-Fernández, R. (2015). Escala de valoración para la evaluación del proceso seguido en el trabajo en equipo. Recuperado 3 de febrero de 2016, a partir de https://drive.google.com/file/d/0B6i1VHoHC4oIUFEybkVld19zczA/view

Palés, J., \& Gomar, C. (2010). El Uso De Las Simulaciones En Educación Médica. Teoría de la Educación, Sociedad de la Información, 11(2), 147-169. Recuperado a partir de http://www.ub.edu/medicina_unitateducaciomedica/documentos/Lus de les simulacions en educacio medica.pdf

Pérez-Pueyo, A., Hortigüela, D., Hernando-Garijo, A., \& Barba-Martín, J. (2013). La coevaluación intragrupal y el reparto de notas bajo un proceso de evaluación formativa. Red Interuniversitaria de Evaluación formativa en Docencia 
Universitaria. Repertorio de «buenas prácticas» en docencia universitaria., 1-7. Recuperado a partir de

https://www.researchgate.net/profile/David_Hortigueela_Alcala/publication/2810 06568_La_coevaluacin_intragrupal_y_el_reparto_de_notas/links/55d12aec08ae6a 881385ea7d.pdf

Valverde, J., Revuelta, F. I., \& Fernández, M. R. (2012). Modelos de evaluación por competencias a través de un sistema de gestión de aprendizaje. Revista Iberoamericana de educación, 60(60), 51-62. Recuperado a partir de http://dialnet.unirioja.es/servlet/articulo?codigo=4772438\&info=resumen\&idioma $\underline{=E N G}$

Wesselink, R., Lans, T., Mulder, M., y Biemans, H. (2003). Competence-based education. An example from vocational practice. En Research Network on Vocational Education and Training (VETNET) at the European Conference on Educational Research (ECER) in Hamburg, 17-20 September 2003 (pp. 1-5). Recuperado a partir de http://www.leeds.ac.uk/educol/documents/00003240.htm 